\title{
A Numerical Method for Solving a Class of Nonlinear Second Order Fractional Volterra Integro-Differntial Type of Singularly Perturbed Problems
}

\author{
Muhammed I. Syam and Mohammed Abu Omar* \\ Department of Mathematical Sciences, College of Science, UAE University, Al-Ain 15551, UAE; \\ m.syam@uaeu.ac.ae \\ * Correspondence: 201150326@uaeu.ac.ae
}

Received: 5 February 2018; Accepted: 28 February 2018; Published: 27 March 2018

\begin{abstract}
In this paper, we study a class of fractional nonlinear second order Volterra integro-differential type of singularly perturbed problems with fractional order. We divide the problem into two subproblems. The first subproblems is the reduced problem when $\epsilon=0$. The second subproblems is fractional Volterra integro-differential problem. We use the finite difference method to solve the first problem and the reproducing kernel method to solve the second problem. In addition, we use the pade' approximation. The results show that the proposed analytical method can achieve excellent results in predicting the solutions of such problems. Theoretical results are presented. Numerical results are presented to show the efficiency of the proposed method.
\end{abstract}

Keywords: singularly perturbed volterra integro-differntial; caputo fractional derivative; nonlinear boundary value problem

\section{Introduction}

Volterra integral equations are considered as a type of integral equations. In 1913, Volterra published the first book that talked about Volterra integral equations. In 1884, Volterra began working on integral equations, but his important study was in 1896 . However, the name Volterra integral equation was first called by Lalesco in 1908. Volterra integral equations have many applications in science and engineering such as elasticity, semi-conductors, scattering theory, seismology, heat conduction, metallurgy, fluid flow, chemical reactions, population dynamics, and spread of epidemics [1].

Volterra integral equations have growlingly been recognized as useful tools for problems in science and engineering. In [2], they proposed and examined a spectral Jacobi-collocation approximation for fractional order integro-differntial equations. Ray et al. [3], used the Legendre wavelet method to find the solutions for a system of nonlinear Volterra integro-differntial equations. In [4], they used Lagurre polynomials and the collocation method to solve the pantograph-type Volterra integro-differntial equations under some initial conditions. Yang et al. [5], discussed the blow-up of Volterra integro-differntial equations with a dissipative linear term to show the differences of the solutions. In [6], they solved a non-linear system of higher order Volterra integro-differntial equations using the Single Term Walsh Series (STWS) method. Also in [7], they solved the fractional Fredholem-Volterra integro-differntial equations by the fractional-order functions based on the Bernoulli polynomials. We also indicate the interested reader to [8-16] for more research works on Volterra integro-differntial equations.

In 1904, A German physicist called Ludwig Prandtl was revolutionized fluid dynamics. He noted that the influence of friction is experienced only very near an object moving through a fluid. In [16], he presented the idea of the boundary layer and its significance for drag and streamlining. In his 
paper, Ludwig Prandtl assumed that the impact of friction was to cause the fluid instantly adjacent to the surface to stick to the surface. This boundary-layer notion had been the basis stone for the new fluid dynamics. Schlichting was one of the most famous books on boundary layer theory [17]. The scientific justification of boundary layer theory gave us more general hypothesis to determine asymptotic expansions of the solutions to the complete equations of the motion. Singular perturbation problem was the result of reduced the problem which was then solved by the method of matched asymptotic expansions. In 1946, Friedrichs and Wasow were the first time used the expression "singular perturbation" [18].

The differential equations of the singularly perturbed problem indicated the study of a group of differential equations including an asymptotically small parameter. The singularly perturbed problem is very important to both applied and pure mathematicians, physicists and engineers because of the fact that the solutions exhibit some interesting behavior. For example, the boundary layer, interior layer, and resonance phenomena [19].

There are a lot of applications of the singularly perturbed problem such as the nonlinear problems of plates and shells by means of the singular perturbation method [20]. Petar discussed typical applications of singular perturbation techniques to control problems in the last fifteen years [21]. Kokotovic et al., showed results on singular perturbations surveyed as a tool for model order reduction and separation of time scales in control system design [22], Ghorbel and Spong, reviewed results of integral manifolds of singularly perturbed non-linear differential equations and outlined the basic elements of the integral manifold method in the context of control system design [23]. Fridman, studied the $H_{\infty}$ control problem for an affine nonlinear singularly perturbed system [24]. Fridman, studied the infinite horizon nonlinear quadratic optimal control problem for a singularly perturbed system [25]. Several other techniques to solve such problems are presented in [26-31].

In this paper, we consider the following class of fractional nonlinear second order Volterra integro-differntial type of singularly perturbed problems of the form

$$
\epsilon D^{\alpha} y+u(x, y) y^{\prime}+\int_{0}^{x} K(x, t) v(t, y) d t=f(x), x \in(0,1), 1<\alpha \leq 2
$$

subject to

$$
y(0)=y_{0}, y(1)=y_{1}
$$

where $\epsilon>0$ is a small positive parameter, $y_{0}$ and $y_{1}$ are constants, and $K(x, t)$ and $f(x)$ are smooth functions. The derivative which we use in this paper is in the Caputo sense. We organize our paper as follows. In Section 2, we present some preliminaries and the reproducing kernel (RKM) method which we use in this paper. In Section 3, we present some analytical results. In Section 4, we present the proposed method. Some numerical results are presented in Section 5. We end this paper by conclusions which presented in Section 6.

\section{Reproducing Kernel Method}

In this section, we present some preliminaries and RKM which we use in this paper.

Definition 1. Let $\alpha>0$ and $x$ be a positive real number. Then, the Riemann-Liouville fractional integral of order $\alpha$ is given by

$$
I^{\alpha} y(x)=\frac{1}{\Gamma(\alpha)} \int_{0}^{x} y(\zeta)(x-\zeta)^{\alpha-1} d \zeta
$$

where $\Gamma$ is the Euler gamma function.

Some of its properties are given as follows: 
- $I^{0} y(x)=y(x)$

- $\quad I^{\alpha}(a y(x)+b y(x))=a I^{\alpha} y(x)+b I^{\alpha} y(x)$ where $a$ and $b$ are constants.

- If $y(x)$ is continuous on $[0, \infty)$, then $I^{\alpha} I^{\beta} y(x)=I^{\beta} I^{\alpha} y(x)=I^{\alpha+\beta} y(x)$ for positive real numbers $\alpha$ and $\beta$.

Definition 2. Let $\alpha>0$ and $x$ be a positive real number. Then, the Caputo derivative of order $\alpha$ is given by

$$
D^{\alpha} y(x)=\left\{\begin{array}{cc}
\frac{1}{\Gamma(n-\alpha)} \int_{0}^{x} \frac{y^{(n)}(\zeta)}{(x-\zeta)^{\alpha-n+1}} d \zeta, & n-1<\alpha<n \in \mathbb{N}, \\
y^{(\alpha)}(x), & \alpha \in \mathbb{N}
\end{array}\right\}
$$

Some of its properties are given as follows:

- $D^{\alpha} x^{\zeta}=\frac{\Gamma(\zeta+1)}{\Gamma(\zeta-\alpha+1)} x^{\zeta-\alpha}$ where $\zeta$ is a real number grater than -1 ,

- $D^{\alpha} c=0$ where $c$ is constant,

- $D^{\alpha}(a y(x)+b y(x))=a D^{\alpha} y(x)+b D^{\alpha} y(x)$ where $a$ and $b$ are constants,

- $\quad$ For $\alpha>0$ and $x$ is a positive real number, $D^{\alpha} I^{\alpha} y(x)=y(x)$,

- $\quad I^{\alpha} D^{\alpha} y(x)=y(x)-\sum_{i=0}^{n-1} \frac{\left(D^{\alpha-i+1} y(0)\right) x^{\alpha-i+1}}{\Gamma(\alpha-i)}, n-1<\alpha \leq n \in \mathbb{N}$.

For more details, see [32,33].

Definition 3. Let $E$ be a nonempty abstract set. A function $M: E \times E \rightarrow C$ is a reproducing kernel of the Hilbert space $H$ if and only if

- $\quad M(., x) \in H$ for all $x \in E$,

- $\quad(\phi(),. M(., x))=\phi(x)$ for all $x \in E$ and $\phi \in H$.

The second condition is called the reproducing property and a Hilbert space which possesses a reproducing kernel is called a reproducing kernel Hilbert space.

Consider the second order nonlinear fractional equation of the form

$$
D^{\alpha} y+g(x, y) y^{\prime}=0, x \in[0,1], 1<\alpha \leq 2
$$

subject to

$$
y(0)=\theta, y(1)=\phi
$$

where $\theta$ and $\phi$ are constants. First, we study the linear case when $g(y)=a(x)$. To homogenize the initial condition, we assume $u=y-\phi x-\theta(1-x)$. Thus, Equations (3) and (4) can be rewritten as

$$
D^{\alpha} u+a(x) u^{\prime}=(-\phi+\theta) a(x)=h(x), x \in[0,1], 0<\alpha \leq 1
$$

subject to

$$
u(0)=0, u(1)=0 .
$$

In order to solve the linear Equations (5) and (6), we construct the kernel Hilbert spaces $W_{2}^{1}[0,1]$ and $W_{2}^{3}[0,1]$ in which every function satisfies the initial condition (6). Let

$$
W_{2}^{1}[0,1]=\left\{u(s): u \text { is absolutely continuous real value function, } u^{\prime} \in L^{2}[0,1]\right\} .
$$


The inner product in $W_{2}^{1}[0,1]$ is defined as

$$
(u(y), v(y))_{W_{2}^{1}[0,1]}=u(0) v(0)+\int_{0}^{1} u^{\prime}(y) v^{\prime}(y) d y,
$$

and the norm $\|u\|_{W_{2}^{1}[0,1]}$ is given by

$$
\|u\|_{W_{2}^{1}[0,1]}=\sqrt{(u(y), u(y))_{W_{2}^{1}[0,1]}}
$$

where $u, v \in W_{2}^{1}[0,1]$.

Theorem 1. The space $W_{2}^{1}[0,1]$ is a reproducing kernel Hilbert space, i.e.; there exists $R(s, y) \in W_{2}^{1}[0,1]$ and its second partial derivative with respect to y exists such that for any $u \in W_{2}^{1}[0,1]$ and each fixed $y, s \in[0,1]$, we have

$$
(u(y), R(s, y))_{\mathrm{W}_{2}^{1}[0,1]}=u(s)
$$

In this case, $R(s, y)$ is given by

$$
R(s, y)=\left\{\begin{array}{rr}
1+y, & y \leq s \\
1+s, & y>s
\end{array}\right\}
$$

Proof. Using the integration by parts, one can get

$$
\begin{aligned}
(u(y), R(s, y))_{W_{2}^{1}[0,1]} & =u(0) R(s, 0)+\int_{0}^{1} u^{\prime}(y) \frac{\partial R}{\partial y}(s, y) d y \\
& =u(0) R(s, 0)+u(1) \frac{\partial R}{\partial y}(s, 1)-u(0) \frac{\partial R}{\partial y}(s, 0)-\int_{0}^{1} u(y) \frac{\partial^{2} R}{\partial y^{2}}(s, y) d y .
\end{aligned}
$$

Since $R(s, y)$ is a reproducing kernel of $W_{2}^{1}[0,1]$,

$$
(u(y), R(s, y))_{\mathrm{W}_{2}^{1}[0,1]}=u(s)
$$

which implies that

$$
\begin{aligned}
& -\frac{\partial^{2} R}{\partial y^{2}}(s, y)=\delta(y-s), \\
& R(s, 0)-\frac{\partial R}{\partial y}(s, 0)=0,
\end{aligned}
$$

and

$$
\frac{\partial R}{\partial y}(s, 1)=0 .
$$

Since the characteristic equation of $-\frac{\partial^{2} R}{\partial y^{2}}(s, y)=\delta(y-s)$ is $\lambda^{2}=0$ and its characteristic value is $\lambda=0$ with 2 multiplicity roots, we write $R(s, y)$ as

$$
R(s, y)=\left\{\begin{array}{ll}
c_{0}(s)+c_{1}(s) y, & y \leq s \\
d_{0}(s)+d_{1}(s) y, & y>s
\end{array} .\right.
$$


Since $\frac{\partial^{2} R}{\partial y^{2}}(s, y)=-\delta(y-s)$, we have

$$
\begin{aligned}
R(s, s+0)-R(s, s+0) & =0, \\
\frac{\partial R}{\partial y}(s, s+0)-\frac{\partial R}{\partial y}(s, s+0) & =-1 .
\end{aligned}
$$

Using Conditions (8)-(11), we get the following system of equations

$$
\begin{aligned}
c_{0}(s)-c_{1}(s) & =0, \\
d_{1}(s) & =0, \\
c_{0}(s)+c_{1}(s) s & =d_{0}(s)+d_{1}(s) s, \\
d_{1}(s)-c_{1}(s) & =-1,
\end{aligned}
$$

which implies that

$$
c_{0}(s)=1, c_{1}(s)=1, d_{0}(s)=1+s, d_{1}(s)=0 .
$$

Next, we study the space $W_{2}^{3}[0,1]$. Let

$$
\begin{aligned}
W_{2}^{3}[0,1] & =\{f(s): f \text { is absolutely continuous real value functions, } \\
f, f^{\prime}, f^{\prime \prime}, f^{\prime \prime \prime} & \left.\in L^{2}[0,1], f(0)=0, f(1)=0\right\} .
\end{aligned}
$$

The inner product in $W_{2}^{3}[0,1]$ is defined as

$$
(u(y), v(y))_{W_{2}^{3}[0,1]}=u(0) v(0)+u^{\prime}(0) v^{\prime}(0)+u(1) v(1)+u^{\prime}(1) v^{\prime}(1)+\int_{0}^{1} u^{(3)}(y) v^{(3)}(y) d y,
$$

and the norm $\|u\|_{W_{2}^{3}[0,1]}$ is given by

$$
\|u\|_{W_{2}^{3}[0,1]}=\sqrt{(u(y), u(y))_{W_{2}^{3}[0,1]}}
$$

where $u, v \in W_{2}^{3}[0,1]$.

Theorem 2. The space $W_{2}^{3}[0,1]$ is a reproducing kernel Hilbert space, i.e.; there exists $K(s, y) \in W_{2}^{3}[0,1]$ which has its six partial derivative with respect to $y$ such that for any $u \in W_{2}^{3}[0,1]$ and each fixed $y, s \in[0,1]$, we have

$$
(u(y), K(s, y))_{W_{2}^{3}[0,1]}=u(s)
$$

In this case, $K(s, y)$ is given by

$$
K(s, y)=\left\{\begin{array}{cc}
\sum_{i=0}^{5} c_{i}(s) y^{i}, & y \leq s \\
\sum_{i=0}^{5} d_{i}(s) y^{i}, & y>s
\end{array}\right\}
$$


where

$$
\begin{aligned}
& c_{0}=0, c_{1}=0, c_{2}=\frac{1}{120}\left(5 s^{4}-111 s^{2}-10 s^{3}-s^{5}\right), \\
& c_{3}=0, c_{4}=-\frac{s}{24}, c_{5}=\frac{1}{120}\left(1+s^{5}\right), \\
& d_{0}=\frac{s^{5}}{120}, d_{1}=-\frac{s^{4}}{24}, d_{2}=\frac{1}{120}\left(5 s^{4}-111 s^{2}-s^{5}\right), d_{3}=-\frac{s^{2}}{12}, \\
& d_{3}=-\frac{s^{2}}{12}, d_{4}=0, d_{5}=\frac{s^{2}}{120} .
\end{aligned}
$$

Proof. Using integration by parts, one can get

$$
\begin{aligned}
(u(y), K(s, y))_{W_{2}^{3}[0,1]=} & u(0) K(s, 0)+u(1) K(s, 1)+u^{\prime}(0) K_{y}(s, 0)+u^{\prime}(1) K_{y}(s, 1) \\
& +u^{\prime \prime}(1) K_{y y y}(s, 1)-u^{\prime \prime}(0) K_{y y y}(s, 0)-u^{\prime}(1) \frac{\partial^{4} K}{\partial y^{4}}(s, 1)+u^{\prime}(0) \frac{\partial^{4} K}{\partial y^{4}}(s, 0) \\
& +u(1) \frac{\partial^{5} K}{\partial y^{5}}(s, 1)-u(0) \frac{\partial^{5} K}{\partial y^{5}}(s, 0)+\int_{0}^{1} u(y) \frac{\partial^{6} K}{\partial y^{6}}(s, y) d y .
\end{aligned}
$$

Since $u(y)$ and $K(s, y) \in W_{2}^{3}[0,1]$,

$$
u(0)=0, u(1)=0
$$

and

$$
K(s, 0)=0, K(s, 1)=0 .
$$

Thus,

$$
\begin{aligned}
(u(y), K(s, y))_{\mathrm{W}_{2}^{3}[0,1]}= & u^{\prime}(0) K_{y}(s, 0)+u^{\prime}(1) K_{y}(s, 1)+u^{\prime \prime}(1) K_{y y y}(s, 1)-u^{\prime \prime}(0) K_{y y y}(s, 0) \\
& -u^{\prime}(1) \frac{\partial^{4} K}{\partial y^{4}}(s, 1)+u^{\prime}(0) \frac{\partial^{4} K}{\partial y^{4}}(s, 0)+\int_{0}^{1} u(y) \frac{\partial^{6} K}{\partial y^{6}}(s, y) d y .
\end{aligned}
$$

Since $K(s, y)$ is a reproducing kernel of $W_{2}^{3}[0,1]$,

$$
(u(y), K(s, y))_{\mathrm{W}_{2}^{3}[0,1]}=u(s)
$$

which implies that

$$
\frac{\partial^{6} K}{\partial y^{6}}(s, y)=\delta(y-s)
$$

where $\delta$ is the dirac-delta function and

$$
\begin{aligned}
K(s, 1)+\frac{\partial^{5} K}{\partial y^{5}}(s, 0) & =0, \\
K_{y}(s, 1)-\frac{\partial^{4} K}{\partial y^{4}}(s, 1) & =0, \\
K_{y y y}(s, 1) & =0, \\
K_{y y y}(s, 0) & =0 .
\end{aligned}
$$

Since the characteristic equation of $\frac{\partial^{6} K}{\partial y^{6}}(s, y)=\delta(s-y)$ is $\lambda^{6}=0$ and its characteristic value is $\lambda=0$ with 6 multiplicity roots, we write $K(s, y)$ as 


$$
K(s, y)=\left\{\begin{array}{cc}
\sum_{i=0}^{5} c_{i}(s) y^{i}, & y \leq s \\
\sum_{i=0}^{5} d_{i}(s) y^{i}, & y>s
\end{array}\right\} .
$$

Since $\frac{\partial^{5} K}{\partial y^{5}}(s, y)=\delta(s-y)$, we have

$$
\frac{\partial^{m} K}{\partial y^{m}}(s, s+0)=\frac{\partial^{m} K}{\partial y^{m}}(s, s-0), m=0,1,2,3,4
$$

On the other hand, integrating $\frac{\partial^{5} K}{\partial y^{5}}(s, y)=\delta(s-y)$ from $s-\epsilon$ to $s+\epsilon$ with respect to $y$ and letting $\epsilon \rightarrow 0$ to get

$$
\frac{\partial^{5} K}{\partial y^{5}}(s, s+0)-\frac{\partial^{5} K}{\partial y^{5}}(s, s-0)=-1 .
$$

Using the Conditions 13 and 15-20, we get the following system of equations

$$
\begin{aligned}
& c_{0}(s)=0, c_{1}(s)=0, c_{3}(s)=0, \\
& 6 d_{3}(s)+24 d_{4}(s)+60 d_{5}(s)=0, \sum_{i=0}^{5} d_{i}(s)+120 d_{5}(s)=0, \\
& \sum_{i=0}^{3} c_{i}(s) s^{i}=\sum_{i=0}^{3} d_{i}(s) s^{i}, \\
& \sum_{i=1}^{5} c_{i}(s) s^{i}=\sum_{i=1}^{5} d_{i}(s) s^{i}, \\
& \sum_{i=1}^{5} i c_{i}(s) s^{i-1}=\sum_{i=i}^{5} i d_{i}(s) s^{i-1}, \\
& \sum_{i=2}^{5} i(i-1) c_{i}(s) s^{i-2}=\sum_{i=1}^{5} i(i-1) d_{i}(s) s^{i-2}, \\
& \sum_{i=3}^{5} i(i-1)(i-2) c_{i}(s) s^{i-3}=\sum_{i=3}^{5} i(i-1)(i-2) d_{i}(s) s^{i-3}, \\
& \sum_{i=4}^{5} i(i-1)(i-2)(i-3) c_{i}(s) s^{i-4}=\sum_{i=4}^{5} i(i-1)(i-2)(i-3) d_{i}(s) s^{i-4}, \\
& 5 ! d_{5}(s)-5 ! c_{5}(s)=-1 .
\end{aligned}
$$

We solved the last system using Mathematica to get

$$
\begin{aligned}
& c_{0}=0, c_{1}=0, c_{2}=\frac{1}{120}\left(5 s^{4}-111 s^{2}-10 s^{3}-s^{5}\right), \\
& c_{3}=0, c_{4}=-\frac{s}{24}, c_{5}=\frac{1}{120}\left(1+s^{5}\right), \\
& d_{0}=\frac{s^{5}}{120}, d_{1}=-\frac{s^{4}}{24}, d_{2}=\frac{1}{120}\left(5 s^{4}-111 s^{2}-s^{5}\right), d_{3}=-\frac{s^{2}}{12} \\
& d_{3}=-\frac{s^{2}}{12}, d_{4}=0, d_{5}=\frac{s^{2}}{120} .
\end{aligned}
$$


Now, we present how to solve Equations (5) and (6). Let

$$
\sigma_{i}(s)=R\left(s_{i}, s\right)
$$

for $i=1,2, \ldots$ where $\left\{s_{i}\right\}_{i=1}^{\infty}$ is dense on $[0,1]$. It is clear that $L: W_{2}^{3}[0,1] \rightarrow W_{2}^{1}[0,1]$ is bounded linear operator. Let

$$
\psi_{i}(s)=L^{*} \sigma_{i}(s)
$$

where $L\left(\sigma_{i}(s)\right)=D^{\alpha} \sigma_{i}(s)+a(s) \sigma_{i}(s)$ and $L^{*}$ is the adjoint operator of $L$. Using Gram-Schmidt orthonormalization to generate orthonormal set of functions $\left\{\bar{\psi}_{i}(s)\right\}_{i=1}^{\infty}$ where

$$
\bar{\psi}_{i}(s)=\sum_{j=1}^{i} \alpha_{i j} \psi_{j}(s)
$$

and $\alpha_{i j}$ are coefficients of Gram-Schmidt orthonormalization. In the next theorem, we show the existence of the solution of Equations (5) and (6).

Theorem 3. If $\left\{s_{i}\right\}_{i=1}^{\infty}$ is dense on $[0,1]$, then

$$
u(s)=\sum_{i=1}^{\infty} \sum_{j=1}^{i} \alpha_{i j} h\left(s_{j}\right) \bar{\psi}_{i}(s) .
$$

Proof. First, we want to prove that $\left\{\psi_{i}(s)\right\}_{i=1}^{\infty}$ is complete system of $W_{2}^{3}[0,1]$ and $\psi_{i}(s)=L\left(K\left(s, s_{i}\right)\right)$. It is clear that $\psi_{i}(s) \in W_{2}^{3}[0,1]$ for $i=1,2, \ldots$. Simple calculations imply that

$$
\begin{aligned}
\psi_{i}(s) & =L^{*} \sigma_{i}(s)=\left(L^{*} \sigma_{i}(s), K(s, y)\right)_{W_{2}^{3}[0,1]} \\
& =\left(\sigma_{i}(s), L(K(s, y))\right)_{W_{2}^{3}[0,1]}=L\left(K\left(s, s_{i}\right)\right)
\end{aligned}
$$

For each fixed $u(s) \in W_{2}^{3}[0,1]$, let

$$
\left(u(s), \psi_{i}(s)\right)_{W_{2}^{3}[0,1]}=0, i=1,2, \ldots
$$

Then

$$
\begin{aligned}
\left(u(s), \psi_{i}(s)\right)_{W_{2}^{3}[0,1]} & =\left(u(s), L^{*} \sigma_{i}(s)\right)_{W_{2}^{3}[0,1]} \\
& =\left(L f(s), \sigma_{i}(s)\right)_{W_{2}^{3}[0,1]} \\
& =L u\left(s_{i}\right)=0 .
\end{aligned}
$$

Since $\left\{s_{i}\right\}_{i=1}^{\infty}$ is dense on $[0,1], L u(s)=0$. Since $L^{-1}$ exists, $u(s)=0$. Thus, $\left\{\psi_{i}(s)\right\}_{i=1}^{\infty}$ is the complete system of $W_{2}^{3}[0,1]$. 
Second, we prove Equation (22). Simple calculations imply that

$$
\begin{aligned}
u(s) & =\sum_{i=1}^{\infty}\left(u(s), \bar{\psi}_{i}(s)\right)_{\mathrm{W}_{2}^{3}[0,1]} \bar{\psi}_{i}(s) \\
& =\sum_{i=1}^{\infty} \sum_{j=1}^{i} \alpha_{i j}\left(u(s), L^{*}\left(K\left(s, s_{j}\right)\right)\right)_{\mathrm{W}_{2}^{3}[0,1]} \bar{\psi}_{i}(s) \\
& =\sum_{i=1}^{\infty} \sum_{j=1}^{i} \alpha_{i j}\left(L f(s), K\left(s, s_{j}\right)\right)_{\mathrm{W}_{2}^{3}[0,1]} \bar{\psi}_{i}(s) \\
& =\sum_{i=1}^{\infty} \sum_{j=1}^{i} \alpha_{i j}\left(c, K\left(s, s_{j}\right)\right)_{W_{2}^{3}[0,1]} \bar{\psi}_{i}(s) \\
& =\sum_{i=1}^{\infty} \sum_{j=1}^{i} \alpha_{i j} h\left(s_{j}\right) \bar{\psi}_{i}(s) .
\end{aligned}
$$

Let the approximate solution of Equations (5) and (6) be given by

$$
u_{N}(s)=\sum_{i=1}^{N} \sum_{j=1}^{i} \alpha_{i j} h\left(s_{j}\right) \bar{\psi}_{i}(s) .
$$

In the next theorem, we show the uniformly convergence of the $\left\{\frac{d^{m} f_{N}(s)}{d s^{m}}\right\}_{N=1}^{\infty}$ to $\frac{d f(s)}{d s}$ for $m=0,1,2$.

Theorem 4. If $u(s)$ and $u_{N}(s)$ are given as in (22) and (23), then $\left\{\frac{d^{m} u_{N}(s)}{d s^{m}}\right\}_{N=1}^{\infty}$ converges uniformly to $\frac{d^{m} u(s)}{d s^{m}}$ for $m=0,1,2$.

Proof. First, we prove the theorem for $m=0$. For any $s \in[0,1]$,

$$
\begin{aligned}
\left\|u(s)-u_{N}(s)\right\|_{W_{2}^{3}[0,1]}^{2} & =\left(u(s)-u_{N}(s), u(s)-u_{N}(s)\right)_{W_{2}^{3}[0,1]} \\
& =\sum_{i=N+1}^{\infty}\left(\left(u(s), \bar{\psi}_{i}(s)\right)_{W_{2}^{3}[0,1]} \bar{\psi}_{i}(s),\left(u(s), \bar{\psi}_{i}(s)\right)_{W_{2}^{3}[0,1]} \bar{\psi}_{i}(s)\right)_{W_{2}^{3}[0,1]} \\
& =\sum_{i=N+1}^{\infty}\left(u(s), \bar{\psi}_{i}(s)\right)_{W_{2}^{3}[0,1]}^{2} .
\end{aligned}
$$

Thus,

$$
\operatorname{Sup}_{s \in[0,1]}\left\|u(s)-u_{N}(s)\right\|_{W_{2}^{3}[0,1]}^{2}=\operatorname{Sup}_{s \in[0,1]} \sum_{i=N+1}^{\infty}\left(u(s), \bar{\psi}_{i}(s)\right)_{\mathrm{W}_{2}^{3}[0,1]}^{2} .
$$

From Theorem (4), one can see that $\sum_{i=1}^{\infty}\left(u(s), \bar{\psi}_{i}(s)\right)_{W_{2}^{3}[0,1]} \bar{\psi}_{i}(s)$ converges uniformly to $u(s)$. Thus,

$$
\operatorname{Lim}_{N \rightarrow \infty} \operatorname{Sup}_{s \in[0,1]}\left\|u(s)-u_{N}(s)\right\|_{W_{2}^{3}[0,1]}=0
$$

which implies that $\left\{u_{N}(s)\right\}_{N=1}^{\infty}$ converges uniformly to $u(s)$.

Second, we prove the uniformly convergence for $m=1,2$. Since $\frac{d^{m} K(s, y)}{d s^{m}}$ is bounded function on $[0,1] \times[0,1]$,

$$
\left\|\frac{d^{m} K(s, y)}{d s^{m}}\right\|_{W_{2}^{3}[0,1]} \leq \chi_{m}, m=1
$$


Thus, for any $s \in[0,1]$,

$$
\begin{aligned}
\left|u^{(m)}(s)-u_{N}^{(m)}(s)\right| & =\left|\left(u(s)-u_{N}(s), \frac{d^{m} K(s, y)}{d s^{m}}\right)_{W_{2}^{3}[0,1]}\right| \\
& \leq\left\|u(s)-u_{N}(s)\right\|_{W_{2}^{3}[0,1]}\left\|\frac{d^{m} K(s, y)}{d s^{m}}\right\|_{W_{2}^{3}[0,1]} \\
& \leq \chi_{m}\left\|u(s)-u_{N}(s)\right\|_{W_{2}^{3}[0,1]} \\
& \leq \chi_{m} \operatorname{Sup}_{s \in[0,1]}\left\|u(s)-u_{N}(s)\right\|_{W_{2}^{3}[0,1]} .
\end{aligned}
$$

Hence,

$$
\operatorname{Sup}_{s \in[0,1]}\left\|u^{(m)}(s)-u_{N}^{(m)}(s)\right\|_{W_{2}^{3}[0,1]} \leq \chi_{m} \operatorname{Sup}_{s \in[0,1]}\left\|u(s)-u_{N}(s)\right\|_{W_{2}^{3}[0,1]}
$$

which implies that

$$
\operatorname{Lim}_{N \rightarrow \infty} \operatorname{Sup}_{s \in[0,1]}\left\|u^{(m)}(s)-u_{N}^{(m)}(s)\right\|_{W_{2}^{3}[0,1]}=0 .
$$

Therefore, $\left\{\frac{d^{m} u_{N}(s)}{d s^{m}}\right\}_{N=1}^{\infty}$ converges uniformly to $\frac{d^{m} u(s)}{d s^{m}}$ for $m=1,2$. Now, we discuss how to solve Equations (3) and (4). Let $\mathcal{L}(y(x))=D^{\alpha} y(x)$ and $N(y(x))=g(x, y) y^{\prime}$ are the linear and nonlinear parts of Problem (3), respectively. We construct the homotopy as follows:

$$
H(y, \lambda)=\mathcal{L}(y(x))+\lambda N(y(x))=0
$$

where $\lambda \in[0,1]$ is an embedding parameter. If $\lambda=0$, we get a linear equation

$$
D^{\alpha} y(x)=0
$$

which implies that $y(x)=\theta$. If $\lambda=1$, we turn out to be Problem (3). Following the Homotopy Perturbation method [34], we expand the solution in term of the Homotopy parameter $\lambda$ as

$$
y=y_{0}+\lambda y_{1}+\lambda^{2} y_{2}+\lambda^{3} y_{3}+\ldots
$$

Substitute Equation (25) into Equation (24) and equating the coefficients of the identical powers of $\lambda$ to get the following system

$$
\begin{array}{ll}
\lambda^{0}: & D^{\alpha} y_{0}(x)=0, y_{0}(0)=\theta \\
\lambda^{1}: & D^{\alpha} y_{1}(x)=-\left.N\left(\sum_{i=0}^{\infty} \lambda^{i} y_{i}(x)\right)\right|_{\lambda=0}, y_{1}(0)=0 \\
\lambda^{2}: & D^{\alpha} y_{2}(x)=-\left.\frac{d N\left(\sum_{i=0}^{\infty} \lambda^{i} y_{i}(x)\right)}{d \lambda}\right|_{\lambda=0}, y_{2}(0) 0 \\
\lambda^{3}: & D^{\alpha} y_{3}(x)=-\left.\frac{d^{2} N\left(\sum_{i=0}^{\infty} \lambda^{i} y_{i}(x)\right)}{d \lambda^{2}}\right|_{\lambda=0}, y_{3}(0)=0 \\
& \vdots \\
\lambda^{k}: & D^{\alpha} y_{k}(x)=-\left.\frac{d^{k-1} N\left(\sum_{i=0}^{\infty} \lambda^{i} y_{i}(x)\right)}{d \lambda^{k-1}}\right|_{\lambda=0}, y_{k}(0)=0 .
\end{array}
$$


To solve the above equations, we use the RKM which described above and we obtain

$$
y_{k}(x)=\sum_{i=1}^{\infty} \sum_{j=1}^{i} \alpha_{i j} h_{k}\left(x_{j}\right) \bar{\psi}_{i}(s), k=0,1, \ldots
$$

where

$$
\begin{aligned}
h_{0}(x)= & 0 \\
h_{1}(x)= & -\left.N\left(\sum_{i=0}^{\infty} \lambda^{i} y_{i}(x)\right)\right|_{\lambda=0} \\
& \vdots \\
h_{k}(x)= & -\left.\frac{d^{k-1} N\left(\sum_{i=0}^{\infty} \lambda^{i} y_{i}(x)\right)}{d \lambda^{k-1}}\right|_{\lambda=0}, k>1 .
\end{aligned}
$$

From Equation (26), it is easy to see the solution to Equations (3) and (4) is given by

$$
y(x)=\sum_{k=0}^{\infty} y_{k}(x)=\sum_{k=0}^{\infty}\left(\sum_{i=1}^{\infty} \sum_{j=1}^{i} \alpha_{i j} h_{k}\left(x_{j}\right) \bar{\psi}_{i}(x)\right) .
$$

We approximate the solution of Equations (3) and (4) by

$$
y_{n, m}(x)=\sum_{k=0}^{m}\left(\sum_{i=1}^{n} \sum_{j=1}^{i} \alpha_{i j} h_{k}\left(x_{j}\right) \bar{\psi}_{i}(x)\right) .
$$

\section{Analytical Results}

In this section, we present the maximum principle, the stability theorem, and the uniqueness theorem. Firstly, Equations (1) and (2) is transformed into an equivalent problem as follows:

$$
\begin{gathered}
P y:-\epsilon D^{\alpha} y+u(x, y) y^{\prime}+\int_{0}^{x} K(x, t) v(t, y) d t=f(x), x \in(0,1), 0<\alpha \leq 1, \\
y(0)=y_{0}, y(1)=y_{1} .
\end{gathered}
$$

The following conditions are needed in order to guarantee that Equations (1) and (2) do not have the turning-point problem

$$
\begin{gathered}
-k_{2} \geq u(x, y) \geq-k_{1}, \\
0 \geq v(x, y) \geq-k_{3}, \\
K(x, t) \geq k_{4} \geq 0,
\end{gathered}
$$

for all $x \in[0,1]$, where $k_{1}, k_{2}, k_{3}$, and $k_{4}$ are positive constants and $y \in C^{2}(0,1) \cup C[0,1]$.

Lemma 1. [35] Let $y \in C^{2}[0,1]$ attains its minimum at $x_{0} \in(0,1)$. Then, $y^{\prime}\left(x_{0}\right) \leq 0$ and $D^{\alpha} y\left(x_{0}\right) \geq 0$ for $1<\alpha \leq 2$. 
Theorem 5. (Maximum Principle). Consider the initial value problem (1) and (2) with conditions (31) and (33). Assume that $P y \geq 0$ and $y(0) \geq 0$. then, $y(1) \geq 0$ in $[0,1]$.

Proof. Assume that the conclusion is false, then $\phi(x)<0$ for some $x \in[0,1]$. Then, $y(x)$ has a local minimum at $x_{0}$ for some $x_{0} \in(0,1]$. Simple calculations and using Lemma (1) imply that

$$
\begin{aligned}
P y\left(x_{0}\right) & =-\epsilon D^{\alpha} y\left(x_{0}\right)+u\left(x_{0}, y\right) y^{\prime}\left(x_{0}\right)+\int_{0}^{x_{0}} K\left(x_{0}, t\right) v(t, y) d t \\
& \leq 0 .
\end{aligned}
$$

This is a contradiction. Therefore, $y(x) \geq 0$ in $[0,1]$.

In the next theorem, the stability result is presented.

Theorem 6. (stability result). Consider Equations (1) and (2) with conditions $u=u(x)$ and $v=v(x)$. If $y(x)$ is a smooth function, then

$$
\|y\|=\max \{|y(x)|: x \in[0,1]\} \leq 2 \zeta \max \left\{\left|y_{0}\right|,\left|y_{1}\right|, \max _{x \in[0,1]}|P y|\right\}
$$

where $\zeta=1+\frac{1}{k_{2}}$.

Proof. Following [36], let

$$
K_{0}=\max \left\{\left|y_{0}\right|,\left|y_{1}\right|, \max _{x \in[0,1]}|P y|\right\}=\max \left\{\left|y_{0}\right|,\left|y_{1}\right|, \max _{x \in[0,1]}|f(x)|\right\}
$$

and

$$
s^{ \pm}(x)=2 \zeta K_{0}\left(1-\frac{x}{2}\right) \pm y(x), x \in[0,1] .
$$

Then,

$$
\begin{aligned}
P s^{ \pm}(x) & =-\epsilon D^{\alpha}\left(2 \zeta K_{0}\left(1-\frac{x}{2}\right) \pm y(x)\right)+u(x)\left(2 \zeta K_{0}\left(1-\frac{x}{2}\right) \pm y(x)\right)^{\prime}+\int_{0}^{x} K(x, t) v(t) d t \\
& =-\zeta K_{0} u(x) \pm P y(x)>K_{0} \pm P y(x) \geq 0
\end{aligned}
$$

for all $x \in[0,1]$. Also,

$$
s^{ \pm}(0)=2 \zeta K_{0} \pm y_{0}>K_{0} \pm y_{0} \geq 0, x \in[0,1]
$$

and

$$
s^{ \pm}(1)=\zeta K_{0} \pm y_{1}>K_{0} \pm y_{1} \geq 0, x \in[0,1] .
$$

From Theorem (5), we can see that $s^{ \pm}(x) \geq 0$ for all $x \in[0,1]$. Therefore,

$$
\|y\|=\max \{|y(x)|: x \in[0,1]\} \leq 2 \zeta \max \left\{\left|y_{0}\right|,\left|y_{1}\right|, \max _{x \in[0,1]}|P y|\right\} .
$$

Theorem 7. (Uniqueness Theorem). Consider Equations (1) and (2) under the conditions (31)-(33) with conditions $u=u(x)$ and $v=v(x)$. If $y_{1}$ and $y_{2}$ are two solutions to Equations (1) and (2), then $y_{1}(x)=y_{2}(x)$ for all $x \in[0,1]$. 
Proof. Let $w(x)=y_{1}(x)-y_{2}(x)$. Then,

$$
\begin{gathered}
P w=0, w(0)=0, w(1)=0 \\
P(-w)=0,-w(0)=0,-w(1)=0 .
\end{gathered}
$$

Using Theorem (5), it follows that $w(x) \geq 0$ and $w(x) \leq 0$ for all $x \in[0,1]$ which imply that $y_{1}(x)=y_{2}(x)$ for all $x \in[0,1]$.

\section{Solution Method}

Consider the following of class of nonlinear second order fractional nonlinear Volterra integro-differential type of singularly perturbed problems of the form

$$
-\epsilon D^{\alpha} y+u(x, y) y^{\prime}+\int_{0}^{x} K(x, t) v(t, y) d t=f(x), x \in(0,1), 1<\alpha \leq 2
$$

subject to

$$
y(0)=y_{0}, y(1)=y_{1}
$$

where $\epsilon>0$ is a small positive parameter, $y_{0}$ and $y_{1}$ are constant, and $K(x, t)$ and $f(x)$ are smooth functions. To solve Equations (1) and (2), we use the following steps.

Step 1: A reduced subproblem is obtained by setting $\epsilon=0$ in Equation (1) to get

$$
u\left(x, y_{1}\right) y_{1}^{\prime}+\int_{0}^{x} K(x, t) v\left(t, y_{1}\right) d t=f(x), x \in[0,1]
$$

On most of the interval, the solution of Equation (34) behaves like the solution of Equations (1) and (2). However, there is small interval around $x=0$ in which the solution of problem (1) and (2) does not agree with the solution of problem (1) and (2). To handle this situation, the boundary layer correction subproblem is introduced in step 2.

Step 2: Choose $x=\epsilon^{\frac{1}{\alpha-1}} s$ to get

$$
\begin{aligned}
D^{\alpha} y(x) & =\frac{1}{\Gamma(1-\alpha)} \int_{0}^{x}(x-t)^{1-\alpha} y^{\prime \prime}(t) d t \\
& =\frac{1}{\Gamma(1-\alpha)} \int_{0}^{\epsilon^{\frac{1}{\alpha-1}} s}\left(\epsilon^{\frac{1}{\alpha-1}} s-t\right)^{1-\alpha} y^{\prime \prime}(t) d t \\
& =\frac{\epsilon^{\frac{1-\alpha}{\alpha-1}}}{\Gamma(1-\alpha)} \int_{0}^{\epsilon^{\frac{1}{\alpha-1}} s}\left(s-\frac{t}{\epsilon^{\frac{1}{\alpha-1}}}\right)^{-\alpha} y^{\prime \prime}(t) d t
\end{aligned}
$$

Let $r=\frac{t}{\epsilon^{\frac{1}{\alpha-1}}}$. Then, $d t=\epsilon^{\frac{1}{\alpha-1}} d r$ and

$$
\begin{aligned}
\frac{d y}{d t} & =\frac{d y}{d r} \frac{d r}{d t}=\frac{1}{\epsilon^{\frac{1}{\alpha-1}}} \frac{d y}{d r} \\
\frac{d^{2} y}{d t^{2}} & =\frac{d\left(\frac{d y}{d t}\right)}{d r} \frac{d r}{d t}=\left(\frac{1}{\epsilon^{\frac{1}{\alpha-1}}}\right)^{2} \frac{d y}{d r}
\end{aligned}
$$


Thus,

$$
\begin{aligned}
D^{\alpha} y(x) & =\frac{\epsilon^{\frac{1-\alpha}{\alpha-1}}}{\Gamma(1-\alpha)} \int_{0}^{s}(s-r)^{-\alpha} \frac{1}{\left(\epsilon^{\frac{1}{\alpha-1}}\right)^{2}} \frac{d y}{d r} \epsilon^{\frac{1}{\alpha-1}} d r \\
& =\frac{\epsilon^{\frac{-\alpha}{\alpha-1}}}{\Gamma(1-\alpha)} \int_{0}^{s}(s-r)^{-\alpha} \frac{d y}{d r} d r \\
& =\epsilon^{\frac{-\alpha}{\alpha-1}} D^{\alpha} y(s) .
\end{aligned}
$$

Hence, Equation (1) becomes

$$
-\epsilon \epsilon^{\frac{-\alpha}{\alpha-1}} D^{\alpha} y(s)+\frac{1}{\epsilon^{\frac{1}{\alpha-1}}} u\left(\epsilon^{\frac{1}{\alpha-1}} s, y\right) \frac{d y}{d s}+\int_{0}^{\epsilon^{\frac{1}{\alpha-1}} s} K\left(\epsilon^{\frac{1}{\alpha-1}} s, t\right) v(t, y) d t=f\left(\epsilon^{\frac{1}{\alpha-1}} s\right)
$$

or

$$
-D^{\alpha} y+u\left(\epsilon^{\frac{1}{\alpha-1}} s, y\right) \frac{d y}{d s}+\epsilon^{\frac{1}{\alpha-1}} \int_{0}^{\epsilon^{\frac{1}{\alpha-1}} s} K\left(\epsilon^{\frac{1}{\alpha-1}} s, t\right) v(t, y) d t=\epsilon^{\frac{1}{\alpha-1}} f\left(\epsilon^{\frac{1}{\alpha-1}} s\right) .
$$

Setting $\epsilon=0$ in Equation (36) implies that

$$
-D^{\alpha} y(s)+u(0, y) \frac{d y}{d s}=0 .
$$

Since the solution of the reduced problem in step 1 does not satisfy the initial condition at $x=0$, then the solution of the above equation should satisfy it. This means, its solution has the form $y_{1}(0)+y_{2}(x)$. Substitute

$$
y(x)=y_{1}(0)+y_{2}(x)
$$

in Equation (38) to get the boundary layer correction equation

$$
-D^{\alpha} y_{2}(s)+u\left(0, y_{1}(0)+y_{2}(s)\right) \frac{d y_{2}}{d s}=0 .
$$

The solution of Equation (1) will be expressed in the form as

$$
y(x)=y_{1}(x)+y_{2}\left(\frac{x}{\epsilon^{\frac{1}{\alpha-1}}}\right)
$$

and the initial condition must be satisfied by expression (40). When $x=0$, the condition will be

$$
y_{0}=y(0)=y_{1}(0)+y_{2}(0)
$$

or

$$
y_{2}(0)=y_{0}-y_{1}(0) .
$$

The solution of Equations (1) and (2) can be produced using the RKM as described in the previous section. More details can be found in [36-39].

\section{Numerical Results}

In this section, we present two of our examples to show the efficiency of the proposed method. 
Example 1: Consider the following problem

$$
-\epsilon D^{\frac{3}{2}} y(x)-2 y^{\prime}(x)-\int_{0}^{x} e^{y(t)} d t=x^{2}-2 x-\frac{2}{x-2}, 0 \leq x \leq 1,0<\epsilon<<1,
$$

subject to

$$
y(0)=0, y(1)=0
$$

When $\epsilon=0$, we get

$$
-2 y^{\prime}(x)-\int_{0}^{x} e^{y(t)} d t=x^{2}-2 x-\frac{2}{x-2}, y(1)=0 .
$$

We discretized the interval $[0,1]$ by $x_{i}=i h, h=\frac{1}{n}, n \in N$. Let $y_{k} \approx y\left(x_{k}\right)$ for $k=0: n$. Using the backward finite difference method to approximate $y^{\prime}\left(x_{k}\right)$ and the trapezoidal quadrature to approximate the integral $\int_{0}^{x_{k}} e^{y(t)} d t$, we get

$$
-2 \frac{y_{k}-y_{k-1}}{h}-h \sum_{j=0}^{k-1}\left(e^{y_{j}}+e^{y_{j+1}}\right)=x_{k}^{2}-2 x_{k}-\frac{2}{x_{k}-2}, x_{n}=0 .
$$

Thus, we get the following system

$$
A Y+B e^{Y}=F
$$

where

$$
\left.\begin{array}{c}
A=-\frac{2}{h}\left(\begin{array}{cccccc}
-1 & 1 & 0 & 0 & \cdots & 0 \\
0 & -1 & 1 & 0 & \cdots & 0 \\
\vdots & \ddots & \ddots & \ddots & \ddots & \vdots \\
\vdots & \cdots & 0 & -1 & 1 & 0 \\
\vdots & \cdots & \cdots & 0 & -1 & 1 \\
0 & \cdots & \cdots & \cdots & 0 & -1
\end{array}\right), B=-h\left(\begin{array}{ccccccc}
1 & 1 & 0 & \cdots & 0 & \cdots & 0 \\
1 & 2 & 1 & \ddots & 0 & \cdots & 0 \\
1 & 2 & 1 & \ddots & 0 & \cdots & 0 \\
\vdots & \vdots & \vdots & \ddots & \ddots & \ddots & \vdots \\
\vdots & \vdots & \vdots & \cdots & 2 & 1 & 0 \\
\vdots & \vdots & \vdots & \cdots & 2 & 2 & 1 \\
2 & 2 & 2 & \cdots & 2 & 2 & 2
\end{array}\right), \\
f\left(x_{1}\right) \\
f\left(x_{1}\right) \\
\vdots \\
f\left(x_{n-1}\right) \\
f\left(x_{n}\right)
\end{array}\right), Y=\left(\begin{array}{c}
y_{0} \\
y_{1} \\
\vdots \\
y_{n-2} \\
y_{n}
\end{array}\right)
$$

Using Mathematica, one can see that the solution of the above system for $n=12$ is given in Figure 1. Using the change of variable $x=\epsilon^{2} s$, we get

$$
-D^{\frac{3}{2}} y_{2}(s)-2 \frac{d y_{2}}{d s}=0
$$

subject to

$$
y_{2}(0)=y_{0}-y_{1}(0)=-0.694147, y_{2}^{\prime}(0)=\theta \text {. }
$$


Using the RKM, we get

$$
\begin{aligned}
y_{2}(s) \approx & -0.694147+\frac{2 \theta s}{3}-\frac{2 s^{2}}{\sqrt{\pi}}+\frac{8 \theta s^{5 / 2}}{5}-\frac{32 \theta s^{3}}{9 \sqrt{\pi}} \\
+ & \frac{16 \theta s^{7 / 2}}{7}-\frac{64 \theta s^{4}}{15 \sqrt{\pi}} .
\end{aligned}
$$

Using the Pade' approximation of order [2, 2], we have $\theta=-0.693147$. In Figures $2-4$, we plot the approximate solution for $\epsilon=0.0001,0.00001$, and 0.000001 , respectively. Let

$$
\operatorname{Error}(x)=\left|-\epsilon D^{\frac{3}{2}} y(x)-2 y^{\prime}(x)-\int_{0}^{x} e^{y(t)} d t-\left(x^{2}-2 x-\frac{2}{x-2}\right)\right| .
$$

In Table 1, we present the error for $x=0,0.1, \ldots, 1$ for $\epsilon=0.0001,0.00001$, and 0.000001. In Table 2, we present the computational time when $\epsilon=0.0001,0.00001$, and 0.000001 .

Table 1. The error for $\epsilon=0.0001,0.00001$, and 0.000001 .

\begin{tabular}{cccc}
\hline $\boldsymbol{x}$ & $\boldsymbol{\epsilon}=\mathbf{0 . 0 0 0 1}$ & $\boldsymbol{\epsilon}=\mathbf{0 . 0 0 0 0 1}$ & $\boldsymbol{\epsilon}=\mathbf{0 . 0 0 0 0 0 1}$ \\
\hline 0 & $2.1 \times 10^{-2}$ & $3.6 \times 10^{-3}$ & $2.8 \times 10^{-6}$ \\
0.1 & $1.9 \times 10^{-3}$ & $5.4 \times 10^{-4}$ & $2.3 \times 10^{-7}$ \\
0.2 & $2.3 \times 10^{-5}$ & $2.4 \times 10^{-7}$ & $2.1 \times 10^{-8}$ \\
0.3 & $3.1 \times 10^{-6}$ & $1.4 \times 10^{-7}$ & $1.9 \times 10^{-8}$ \\
0.4 & $1.8 \times 10^{-7}$ & $1.8 \times 10^{-9}$ & $1.5 \times 10^{-11}$ \\
0.5 & $1.5 \times 10^{-9}$ & $1.3 \times 10^{-11}$ & $1.1 \times 10^{-12}$ \\
0.6 & $3.4 \times 10^{-9}$ & $2.2 \times 10^{-12}$ & $2.1 \times 10^{-13}$ \\
0.7 & $3.3 \times 10^{-9}$ & $2.3 \times 10^{-13}$ & $2.2 \times 10^{-14}$ \\
0.8 & $3.1 \times 10^{-11}$ & $1.4 \times 10^{-13}$ & $1.7 \times 10^{-14}$ \\
0.9 & $1.0 \times 10^{-13}$ & $1.5 \times 10^{-14}$ & $1.4 \times 10^{-15}$ \\
1 & $2.1 \times 10^{-15}$ & $2.2 \times 10^{-16}$ & $1.1 \times 10^{-16}$ \\
\hline
\end{tabular}

Table 2. Computational time.

\begin{tabular}{cc}
\hline $\boldsymbol{\epsilon}$ & Computational time \\
\hline 0.0001 & $3.4 S$ \\
0.00001 & $4.6 S$ \\
0.000001 & $6.1 S$ \\
\hline
\end{tabular}

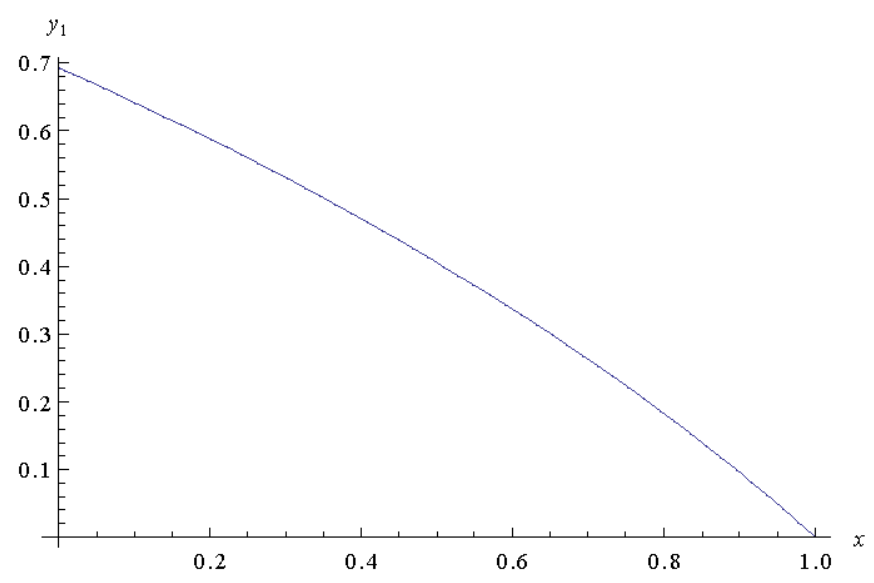

Figure 1. The approximate solution $y_{1}$. 


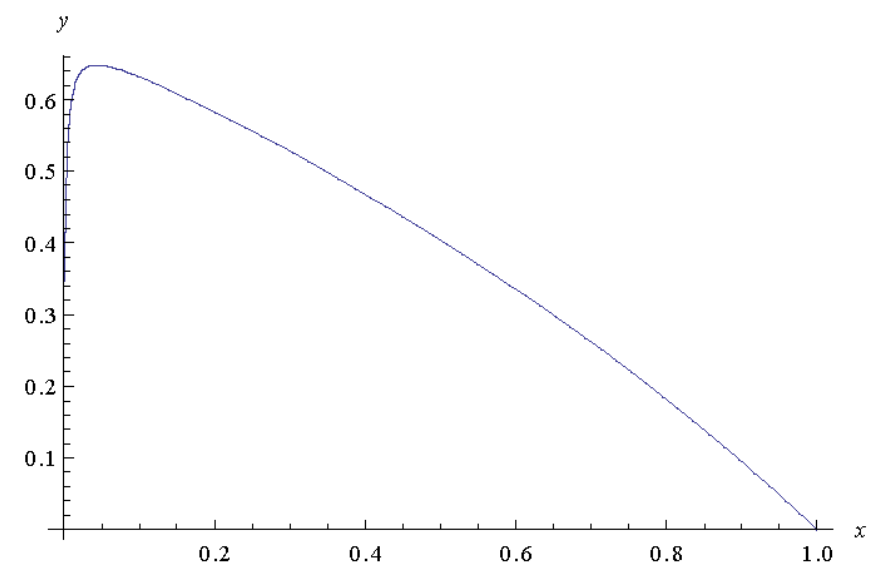

Figure 2. The approximate solution $y$ for $\epsilon=0.0001$.

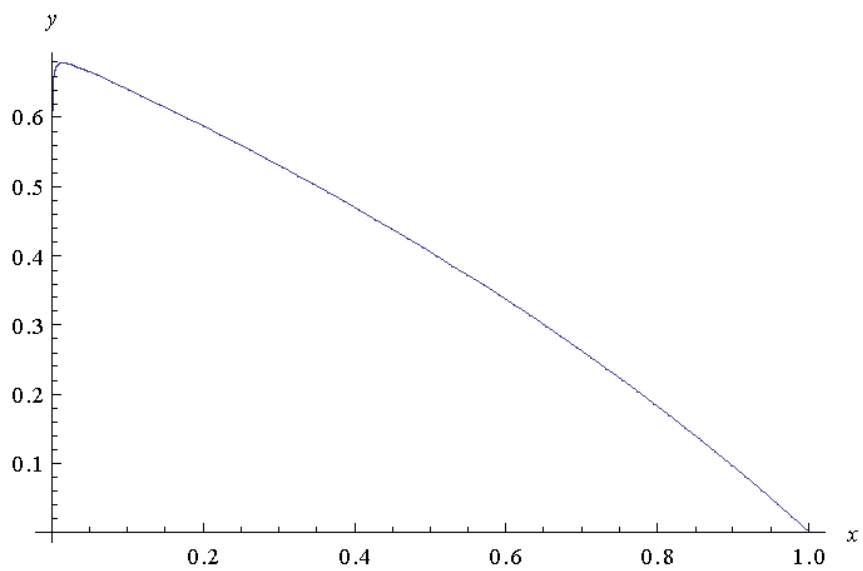

Figure 3. The approximate solution $y$ for $\epsilon=0.00001$.

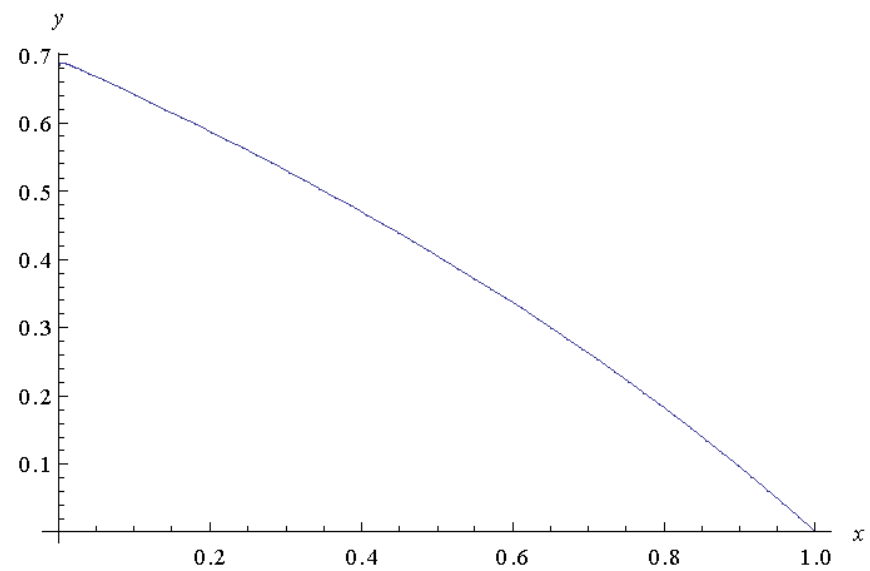

Figure 4. The approximate solution $y$ for $\epsilon=0.000001$.

Example 2: Consider the following problem

$$
-\epsilon D^{\frac{3}{2}} y(x)-y y^{\prime}-\int_{0}^{x}(x-t)^{2} y^{2}(t) d t=f(x), 0 \leq x \leq 1,0<\epsilon<<1
$$


subject to

$$
y(0)=-1, y(1)=6
$$

where

$$
f(x)=-5-x-\frac{25}{3} x^{3}-\frac{5}{6} x^{4}-\frac{x^{5}}{30} .
$$

When $\epsilon=0$, we get

$$
-y y^{\prime}-\int_{0}^{x}(x-t) y^{2}(t) d t=f(x), y(1)=6 .
$$

We discretized the interval $[0,1]$ by $x_{i}=i h, h=\frac{1}{n}, n \in N$. Let $y_{k} \approx y\left(x_{k}\right)$ for $k=0: n$. Using the backward finite difference method to approximate $y^{\prime}\left(x_{k}\right)$ and the trapezoidal quadrature to approximate the integral $\int_{0}^{x_{k}}(x-t) y^{2}(t) d t$, we get

$$
-y_{k} \frac{y_{k}-y_{k-1}}{h}-\frac{h}{2} \sum_{j=0}^{k-1}\left(\left(x_{k}-x_{j+1}\right) y_{j+1}^{2}+\left(x_{k}-x_{j}\right) y_{j}^{2}\right)=f\left(x_{k}\right), y_{n}=6 .
$$

Using Mathematica, one can see that the solution of the above system for $n=12$ is given in Figure 5. Using the change of variable $x=\epsilon^{2} s$, we get

$$
-D^{\frac{3}{2}} y_{2}(s)-\left(y_{2}(s)+5\right) \frac{d y_{2}}{d s}=0
$$

subject to

$$
y_{2}(0)=y_{0}-y_{1}(0)=-6, y_{2}^{\prime}(0)=\theta \text {. }
$$

Using the RKM, we get

$$
y_{2}(s) \approx-6+\theta s-\frac{4 \theta}{3 \sqrt{\pi}} s^{\frac{3}{2}}+\frac{\theta}{2} s^{2}-\frac{8 \theta^{2}}{15 \sqrt{\pi}} s^{\frac{5}{2}}-\frac{7 \theta^{2}}{12} s^{3}+\frac{7 \theta^{3}}{48} s^{4} .
$$

Using the Pade' approximation of order $[2,2]$, we have $\theta=0.0927388622769557$. In Table 3, we present the error for $x=0,0.1, \ldots, 1$ for $\epsilon=0.001,0.0001$, and 0.00001 . In Table 4 , we present the computational time when $\epsilon=0.001,0.0001$, and 0.00001 .

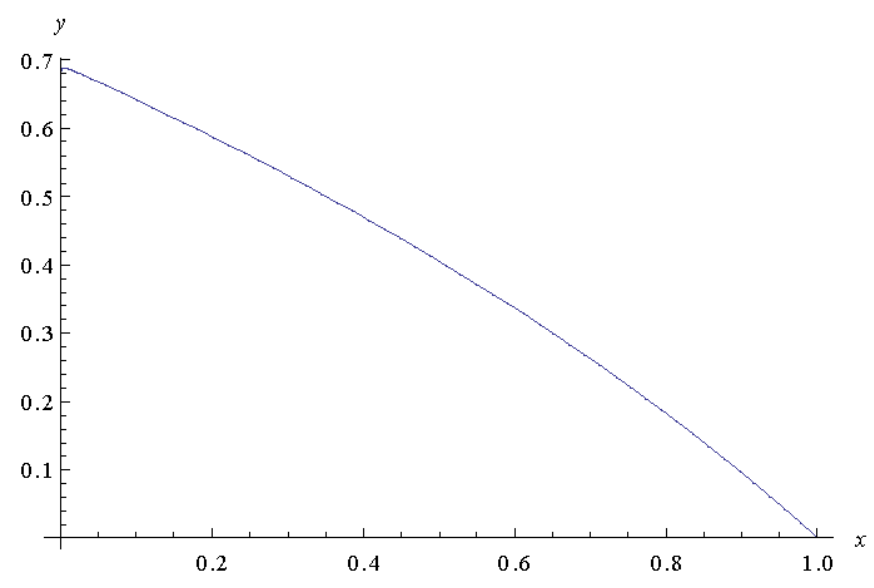

Figure 5. The approximate solution $y_{1}$. 
Table 3. The error for $\epsilon=0.0001,0.00001$, and 0.000001 .

\begin{tabular}{llll}
\hline $\boldsymbol{x}$ & $\boldsymbol{\epsilon}=\mathbf{0 . 0 0 1}$ & $\boldsymbol{\epsilon}=\mathbf{0 . 0 0 0 1}$ & $\boldsymbol{\epsilon}=\mathbf{0 . 0 0 0 0 1}$ \\
\hline 0 & $6.1 \times 10^{-2}$ & $6.6 \times 10^{-3}$ & $7.2 \times 10^{-6}$ \\
0.1 & $5.5 \times 10^{-3}$ & $6.1 \times 10^{-4}$ & $7.1 \times 10^{-7}$ \\
0.2 & $4.8 \times 10^{-5}$ & $4.3 \times 10^{-7}$ & $7.0 \times 10^{-8}$ \\
0.3 & $4.1 \times 10^{-6}$ & $4.1 \times 10^{-7}$ & $5.8 \times 10^{-8}$ \\
0.4 & $3.7 \times 10^{-7}$ & $3.9 \times 10^{-9}$ & $5.6 \times 10^{-11}$ \\
0.5 & $3.4 \times 10^{-9}$ & $3.8 \times 10^{-11}$ & $5.2 \times 10^{-12}$ \\
0.6 & $3.1 \times 10^{-9}$ & $3.4 \times 10^{-12}$ & $5.1 \times 10^{-13}$ \\
0.7 & $2.5 \times 10^{-9}$ & $3.1 \times 10^{-13}$ & $3.4 \times 10^{-14}$ \\
0.8 & $2.3 \times 10^{-11}$ & $2.4 \times 10^{-13}$ & $3.3 \times 10^{-14}$ \\
0.9 & $2.1 \times 10^{-13}$ & $2.2 \times 10^{-14}$ & $2.8 \times 10^{-15}$ \\
1 & $1.9 \times 10^{-15}$ & $2.1 \times 10^{-16}$ & $1.7 \times 10^{-16}$ \\
\hline
\end{tabular}

Table 4. Computational time.

\begin{tabular}{cc}
\hline $\boldsymbol{\epsilon}$ & Computational time \\
\hline 0.001 & $3.5 S$ \\
0.0001 & $4.7 S$ \\
0.00001 & $6.2 S$ \\
\hline
\end{tabular}

Thus,

$$
y(x)=y_{1}(x)+y_{2}\left(\frac{x}{\epsilon^{2}}\right)
$$

In Figures 6-8, we plot the approximate solution for $\epsilon=0.001,0.0001$, and 0.00001 , respectively.

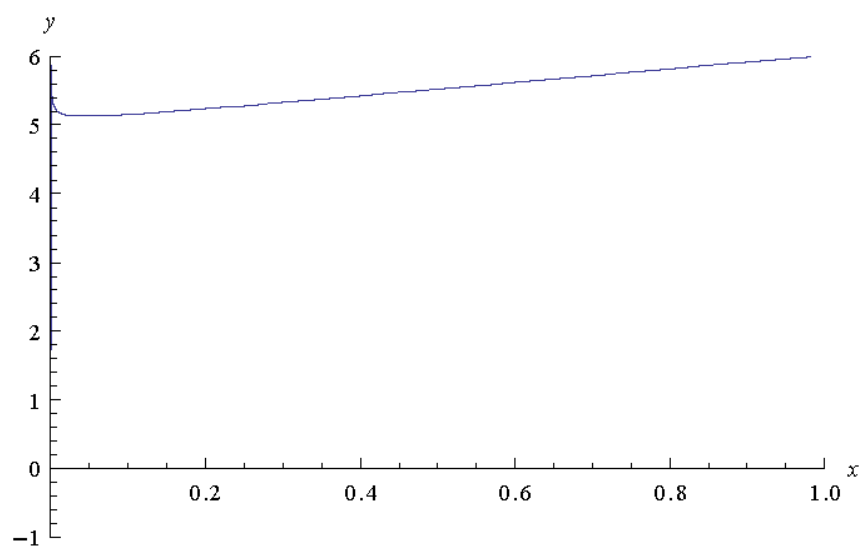

Figure 6. Approximate solution for $\epsilon=0.001$. 


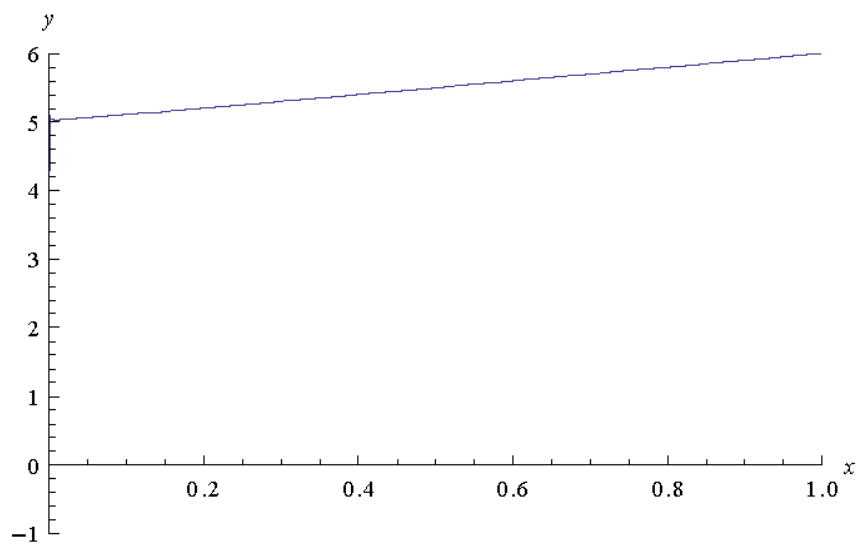

Figure 7. Approximate solution for $\epsilon=0.0001$.

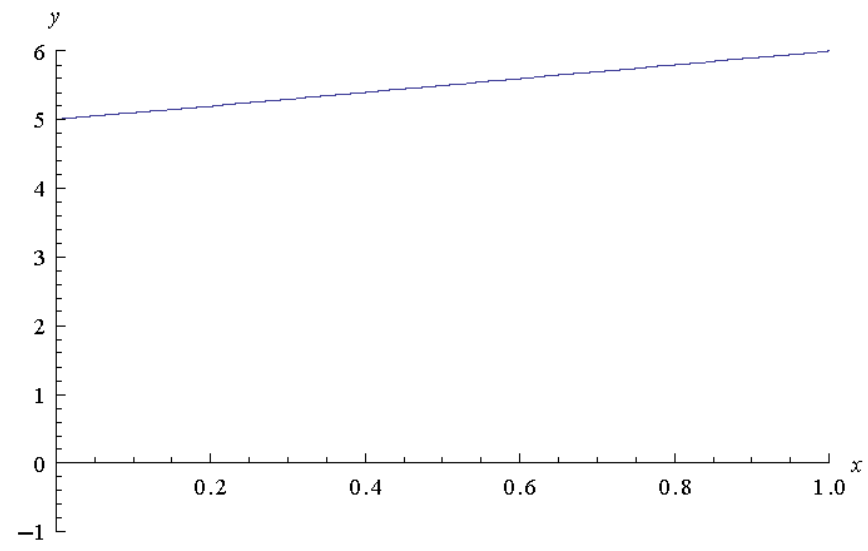

Figure 8. Approximate solution for $\epsilon=0.00001$.

\section{Conclusions}

In this paper, we study a class of fractional nonlinear second order Volterra integro-differential type of singularly perturbed problems with fractional order. We divide the problem into two subproblems. The first subproblem is the reduced problem when $\epsilon=0$. The second subproblem is the second order fractional Volterra integro-differential problem. We use the finite difference method to solve the first subproblem and the reproducing kernel method to solve the second subproblem. The results show that the proposed analytical method can achieve excellent results in predicting the solutions of such problems. Theoretical results are presented. Numerical results are presented to show the efficiency of the proposed method. Figures 1-8 show the efficiency of the proposed method.

Acknowledgments: The authors would like to thank the reviewers for the valuable comments.

Author Contributions: All authors have equal contributionss in all parts of this paper.

Conflicts of Interest: The authors declare no conflict of interest.

\section{References}

1. Tsalyuk, Z.B. Volterra integral equations. J. Sov. Math. 1979, 12, 715-758.

2. Yang, Y.; Chen, Y.; Huang, Y. Spectral-collocation method for fractional Fredholm integro-differntial equations. J. Korean Math. Soc. 2014, 51, 203-224.

3. Sahu, P.K.; Ray, S.S. Legendre wavelets operational method for the numerical solutions of nonlinear Volterra integro-differntial equations system. Appl. Math. Comput. 2015, 256, 715-723. 
4. Yüzbaşı, Ş. Laguerre approach for solving pantograph-type Volterra integro-differntial equations. Appl. Math. Comput. 2014, 232, 1183-1199.

5. Yang, Z.; Tang, T.; Zhang, J. Blowup of Volterra Integro-differntial Equations and Applications to Semi-Linear Volterra Diffusion Equations. Numer. Math. Theory Methods Appl. 2017, 10, 737-759.

6. Sekar, R.C.G.; Murugesan, K. Numerical Solutions of Non-Linear System of Higher Order Volterra Integro-differntial Equations using Generalized STWS Technique. In Differential Equations and Dynamical Systems; Oxford University Press: Oxford, UK, 2017; pp. 1-13.

7. Rahimkhani, P.; Ordokhani, Y.; Babolian, E. Fractional-order Bernoulli functions and their applications in solving fractional Fredholem-Volterra integro-differntial equations. Appl. Numer. Math. 2017, 122, 66-81.

8. Jiang, Y.; Ma, J. Spectral collocation methods for Volterra-integro differential equations with noncompact kernels. J. Comput. Appl. Math. 2013, 244, 115-124.

9. Öztürk, Y.; Gülsu, M. An Operational Matrix Method for Solving a Class of Nonlinear Volterra Integro-differntial Equations by Operational Matrix Method. Int. J. Appl. Comput. Math. 2017, 3, 1-16.

10. Alvandi, A.; Paripour, M. Reproducing kernel method with Taylor expansion for linear Volterra integro-differntial equations. Commun. Numer. Anal. 2017, 2017, 40-49.

11. Tunç, C. New stability and boundedness results to Volterra integro-differntial equations with delay. J. Egypt. Math. Soc. 2016, 24, 210-213.

12. Ma, X.; Huang, C. Spectral collocation method for linear fractional integro-differntial equations. Appl. Math. Model. 2014, 38, 1434-1448.

13. Berenguer, M.I.; Garralda-Guillem, A.I.; Galán, M.R. An approximation method for solving systems of Volterra integro-differntial equations. Appl. Numer. Math. 2013, 67, 126-135.

14. Lovitt, W.V. Linear Integral Equations; Courier Corporation: North Chelmsford, MA, USA, 2014.

15. Song, Y.; Kim, H. The solution of Volterra integral equation of the second kind by using the Elzaki transform. Appl. Math. Sci. 2014, 8, 525-530.

16. Prandtle, L. Uber flussigkeits-bewegung bei kleiner reibung verhandlungen. In Proceedings of the III International Math Congress, Tuebner, Leipzig, 5-7 December 1905; pp. 484-491.

17. Schlichting, H. Boundary-Layer Theory; Mcgraw-Hill: New York, NY, USA, 1979.

18. Friedrichs, K.O.; Wasow, W. Singular perturbations of nonlinear oscillations. Duke Math. J. 1946, 13, 367-381.

19. Abrahamsson, L.R. A prior estimates for solutions of singular perturbations with a turning point. Stud. Appl. Math. 1977, 56, 51-69.

20. Chou, H. Some applications of the singular perturbation method to the bending problems of thin plates and shells. Appl. Math. Mech. 1948, 5, 1449-1457.

21. Kokotović, P.V. Applications of singular perturbation techniques to control problems. SIAM Rev. 1984, 26, 501-550.

22. Kokotovic, P.V.; O'malley, R.E.; Sannuti, P. Singular perturbations and order reduction in control theory-An overview. Automatic 1976, 12, 123-132.

23. Ghorbel, F.; Spong, M.W. Integral manifolds of singularly perturbed systems with application to rigid-link flexible-joint multibody systems. Int. J. Non-Linear Mech. 2000, 35, 133-155.

24. Fridman, E. State-feedback $H_{\infty}$ control of nonlinear singularly perturbed systems. Int. J. Robust Nonlinear Control 2001, 11, 1115-1125.

25. Fridman, E. Exact slow-Fast decomposition of the nonlinear singularly perturbed optimal control problem. Syst. Control Lett. 2000, 40, 121-131.

26. Archibasov, A.A.; Korobeinikov, A.; Sobolev, V.A. Passage to the limit in a singularly perturbed partial integro-differential system. Differ. Equ. 2016, 52, 1115-1122.

27. Archibasov, A.A.; Korobeinikov, A.; Sobolev, V.A. Asymptotic expansions of solutions in a singularly perturbed model of virus evolution. Comput. Math. Math. Phys. 2015, 55, 240-250.

28. Korobeinikov, A.; Archibasov, A.; Sobolev, V. Order reduction for an rna virus evolution model. Math. Biosci. Eng. 2015, 12, 1007-1016

29. Nefedov, N.N.; Nikitin, A.G. Method of differential inequalities for step-like contrast structures in singularly perturbed integro-differential equations in the spatially two-dimensional case. Diff. Equ. 2006, 42, 739, doi:10.1134/S0012266106050132.

30. Nefedov, N.N.; Nikitin, A.G. Boundary and internal layers in the reaction-diffusion problem with a nonlocal inhibitor. Comput. Math. Math. Phys. 2011, 51, 1011, doi:10.1134/S0965542511060157. 
31. Omel'chenko, O.E.; Nefedov, N.N. Boundary-layer solutions to quasilinear integro-differential equations of the second order. Comput. Math. Math. Phys. 2002, 42, 470-482.

32. Kashkari, B.; Syam, M. Fractional-order Legendre operational matrix of fractional integration for solving the Riccati equation with fractional order. Appl. Math. Comput. 2016, 290, 281-291.

33. Syam, M.; Siyyam, H.; Al-Subaihi, I. Tau-Path Following method for Solving the Riccati Equation with Fractional Order. J. Comput. Methods Phys. 2014, 2014, 207916.

34. Ariel, P.D.; Syam, M.I.; Al-Mdallal, Q.M. The extended homotopy perturbation method for the boundary layer flow due to a stretching sheet with partial slip. Int. J. Comput. Math. 2013, 90, 1990-2002.

35. Al-Refai, M. On the Fractional Derivatives at Extreme Points. Electron. J. Qual. Theory Differ. Equ. 2012, 55, 1-5.

36. Al-Mdallal, Q.; Syam, M. An efficient method for solving non-linear singularly perturbed two points boundary-value problems of fractional order. Commun. Nonlinear Sci. Numer. Simul. 2012, 17, 2299-2308.

37. Syam, M.; Attili, B. Numerical solution of singularly perturbed fifth order two point boundary value problem. Appl. Math. Comput. 2005, 170, 1085-1094.

38. Kashkaria, B.; Syam, M. Evolutionary computational intelligence in solving a class of nonlinear Volterra-Fredholm integro-differntial equations. J. Comput.l Appl. Math. 2017, 311, 314-323.

39. Nefedov, N.N.; Nikitin, A.G. The asymptotic method of differential inequalities for singularly perturbed integro-differential equations. Differ. Equ. 2000, 36, 1544-1550, doi:10.1007/BF02757396).

(c) 2018 by the authors. Licensee MDPI, Basel, Switzerland. This article is an open access article distributed under the terms and conditions of the Creative Commons Attribution (CC BY) license (http:// creativecommons.org/licenses/by/4.0/). 